\title{
COLABORACIÓN Y GOBERNANZA PARA EL DESARROLLO TURÍSTICO. ARANJUEZ COMO ESTUDIO DE CASO
}

\author{
Ana Muñoz-Mazón* \\ Universidad Rey Juan Carlos. Madrid. \\ María Velasco González** \\ Universidad Complutense de Madrid
}

\section{RESUMEN}

El artículo se basa en una investigación sobre las posibilidades de crear un órgano de gestión del destino, de carácter mixto, para la gestión turística de Aranjuez. Se argumenta que para elegir el mejor modelo de órgano de gestión turística para un destino es necesario investigar tres categorías: caracterización del turismo, importancia relativa del turismo y madurez colaborativa de los actores turísticos del destino. La conclusión es que no existe un modelo de órgano de gestión mixta que pueda ser implantado en cualquier destino turístico, siendo el contexto específico y sus condiciones los que acaban aconsejando qué tipo de institución de gestión de destino es posible diseñar e implantar ${ }^{1}$.

Palabras clave: Gobernanza, cooperación, organizaciones de gestión de destinos.

\section{Cooperation and governance for tourism development. Aranjuez as a case study}

\section{ABSTRACT}

This article describes the main reflections of an investigation about the possibility to create a destination management organization for the city of Aranjuez. In order to select the best tourism destination organization for a municipal level three categories need to be

Recibido: 20 de julio de 2013

Devuelto para su revisión: 22 de enero de 2014

Aceptado: 28 de abril de 2014

* Departamento Economía de la Empresa. Facultad de Ciencias Sociales. Universidad Rey Juan Carlos. Paseo Artilleros, s/n. 28032 Vicálvaro. MADRID (España).Email: ana.munoz@urjc.es

** Facultad de Ciencias Políticas. Departamento de Ciencia Politica y de la Administración II. Universidad Complutense.Campus de Somosaguas. 28223 Pozuelo. MADRID (España).E-mail: maria.velasco@cps.ucm.es

1 Este artículo tiene su origen en un proyecto de investigación sustentado en un convenio entre el Ayuntamiento de Aranjuez y el CES Felipe II. Las autoras quieren agradecer a los revisores/as de la primera versión del artículo todos sus comentarios. Sus sugerencias han permitido mejorar mucho el presente trabajo. 
investigated: characterization of the type of tourism, relative importance of tourism and collaborative maturity of the tourism stakeholders. The conclusion is that there is not one single model of a public-private management organization that can be implemented directly. The specific context and conditions of tourism destinations determine which type of destination management organization can be designed and implemented.

Key words: Governance, cooperation, Destination Management Organizations.

\section{IMPORTANCIA DE LA COLABORACIÓN PÚBLICO-PRIVADA EN EL TURISMO}

El desarrollo de los destinos turísticos locales supone un proceso en el que una sociedad, manteniendo su propia identidad y su territorio, genera y fortalece sus dinámicas económicas, sociales y culturales, para permitir la articulación de los diferentes componentes del turismo y su puesta a disposición del mercado. Para poder llevar a cabo este proceso es fundamental la participación de todos los agentes o fuerzas turísticas que interactúan dentro de los límites del sistema. Es también imprescindible contar con un proyecto común que combine distintos valores: generación de crecimiento económico, equidad, cambio social y cultural, sostenibilidad medio ambiental, enfoque de género y calidad de vida, entre otros.

En los últimos años se ha pasado de una situación en la que el Estado tenía la responsabilidad plena del bienestar público y las empresas se concentraban en incrementar sus beneficios, independientemente de los intereses de la sociedad, a un mundo en el que el éxito depende de la comunidad de intereses entre las empresas, la sociedad civil y los gobiernos (OMT, 2001).

Teniendo en cuenta a todos los actores, procedentes de diferentes ámbitos y con intereses en ocasiones opuestos, puede haber un gran potencial para poner en marcha políticas de carácter holístico e integral que consigan y ayuden al desarrollo sostenible de un destino. Asimismo, es importante reconocer que, dada la complejidad actual del sistema turístico, los problemas que en el mismo suelen surgir no pueden ser resueltos por un solo agente sino que se precisa de la colaboración de varios. Por ello, cada uno de los interlocutores habrá de sentir que el modelo de destino turístico resultante y la dirección que este tomará en el futuro, es fruto de sus actuaciones conjuntas. Esto significa que la administración pública turística no debería imponer sus políticas sino que tendrían que consensuarlas con el resto de agentes públicos y privados. En este nuevo contexto, la función de autoridad y gobierno tiene que reinventarse y buscar el desarrollo de un papel más estratégico, sustituyendo sus actuaciones tradicionales por otras que fomenten la generación de confianza entre los agentes del sistema turístico (Velasco, 2013).

Relacionado con esta idea de transformación social aparece el término gobernanza, concepto complejo que agrupa diferentes teorías y prácticas cuyo único punto en común es la preocupación por entender que, junto a las formas clásicas de organización social - el mercado para la esfera privada y la jerarquía para el ámbito público - aparecen nuevas formas en las que la cooperación y colaboración son la clave (Bevir, 2011). Los aportes sobre 
las dimensiones de la gobernanza son muy variados y ricos y se sitúan en un continuum de base ideológica que iría desde una posición neoliberal - con teorías y propuestas cuyo objetivo es la reducción o vaciamiento del Estado a favor el funcionamiento del mercado - a una posición neo estatal - con propuestas teóricas y acciones que proponen convertir la gobernanza en una nueva herramienta de gobierno (Torfing et all. 2012). Del conjunto de reflexiones las redes de actores o networks constituyen un objeto de trabajo singular y central que ya acumula propuestas y teorías sustantivas (Börzel, 1998).

La formación de redes y su impacto en la organización social se constituye como otra característica de las nuevas formas de gobernanza. La hipótesis de trabajo es que las redes sociales de colaboración y cooperación entre agentes dentro del sistema, apoyadas por un marco institucional fortalecido, generarán externalidades positivas, lo que producirá un desarrollo que contribuirá de manera decisiva al buen funcionamiento tanto del conjunto del sistema como de los actores que los componen.

En conexión con la idea de formación de redes, se encuentran en la literatura referencia a las ideas de colaboración y cooperación (Bramwell y Lane, 1999; Dredge, 2006; Pavlovich, 2008; Reed, 1999; Scott, Baggio, \& Cooper, 2008). Ambos términos son usados frecuentemente como sinónimos en la literatura de planificación y política turística pero, según Jamal y Getz (1995), sin que se hayan realizado esfuerzos para diferenciar un concepto del otro. Según estos autores, cooperación significaría «trabajar conjuntamente con alguna finalidad», sin atender a criterios más específicos de interdependencia organizacional. La colaboración, sin embargo, ofrece un «proceso dinámico de resolución de problemas derivados de la planificación y de coordinación del desarrollo turístico local» (Jamal y Getz, 1995). De este modo, la teoría de colaboración ofrece amplias posibilidades para la gestión y planificación de destinos turísticos, teniendo en cuenta que cada uno de los agentes interrelacionados mantiene posturas divergentes con respecto al desarrollo turístico local.

La literatura ha evolucionado en las últimas décadas respecto a cómo los acuerdos entre actores pueden llegar a tener un papel determinante en el desarrollo de un destino turístico (Selin, 1999; Selin y Myers, 1998). Estos acuerdos podrían entenderse como situaciones donde existe un interés en compartir recursos (información, dinero, fuerza laboral, etc.) entre dos o más agentes para así solventar un problema o crear una oportunidad que no podría surgir de manera individual (Selin \& Chavez, 1995). Estas formas no tradicionales de análisis han ido ganando presencia en perspectivas que tratan de entender las dinámicas internas de los acuerdos así como las fuerzas externas que facilitan la aparición y el crecimiento de los mismos (Selin, 1999; Dredge y Pforr, 2008).

La naturaleza del proceso de colaboración entre agentes está muy relacionada con la situación que da origen a la estrategia de cooperación. En este sentido, podría ocurrir que los actores de un destino turístico se vieran impulsados a cooperar para gestionar una ayuda o asignación financiera concreta y coyuntural (programa, financiación excepcional, etc.), en cuyo caso la lógica cooperativa estaría limitada en el tiempo y seguiría la meta del proyecto, siguiendo una lógica meramente técnico-económica (Merinero, 2009; Merinero y Pulido, 2009). En estas situaciones, sería muy complicado asegurar que el proceso relacional genera el suficiente capital social como para contribuir eficientemente al desarrollo del destino turístico. A pesar de ello, en ocasiones, estos procesos provocan una experi- 
encia tan positiva que, tras su finalización, los grupos de interés continúan generando procesos de colaboración. Las externalidades, que la situación de trabajo cooperativo ha producido, impulsan a los agentes a provocar relaciones continuadas en el tiempo.

Pero también puede ocurrir que la colaboración se plantee como un mecanismo de desarrollo al servicio de una estrategia local a largo plazo. Para ello se requiere tiempo y un proceso cuya finalidad sea concienciar a todos los agentes, modificar sus comportamientos y lograr el compromiso responsable del mayor número de grupos de interés posible. En este sentido, el proceso de colaboración surge en el momento en el que algún grupo de actores tiene el deseo de cooperar con otro u otros elementos del sistema turístico. Si bien la iniciativa de colaboración puede partir de cualquiera de los actores locales, son las instituciones públicas las tienen la responsabilidad de promover la cooperación entre agentes a través de mecanismos formales o informales. En la actualidad, las instituciones adquieren un papel fundamental en los procesos de desarrollo económico (North, 1990), por tanto, el marco institucional de un destino ha de definir las reglas del juego por las que los actores estratégicos del mismo tendrán que guiarse.

Las instituciones, además de establecer las reglas del juego entre agentes, han de proveer a los distintos actores estratégicos de incentivos que les empujen a la colaboración, tratando de controlar los posibles frenos a la misma que podrían existir en un sistema turístico. En este ámbito de estudio, las limitaciones a la colaboración vendrían definidas por: cuestiones demográficas, si existe escasa densidad de población o lejanía de los grandes núcleos urbanos, se genera poca mano de obra susceptible de ser utilizada para el sistema turístico, situaciones geográficas ofísicas en las que se genera con mayor facilidad una degradación del medio ambiente y un deterioro de recursos que podrían utilizarse para poner en valor productos turísticos, existencia de grupos de presión o poder constituidos sólidamente, los cuales se encuentran fuertemente asentados en un destino e imponen sus propias reglas del juego, dejando un margen muy pequeño para la expresión del interés colectivo, experiencias negativas en otros procesos de cooperación de modo que la fragmentación social que se produce dificulta nuevos procesos de cooperación local y la falta de iniciativas locales y cuestiones de carácter cultural por la no existencia de concienciación social sobre las ventajas que un sistema turístico puede generar en la comunidad local o, incluso, por situaciones de éxodo rural del territorio de forma que la población que se ha quedado en la zona está compuesta únicamente por las personas de mayor edad (Muñoz, 2009).

\section{LAS ENTIDADES DE GESTIÓN MIXTA EN TURISMO: ORGANIZACIONES DE GESTIÓN DE DESTINOS}

La colaboración entre el sector público y el sector privado en turismo es imprescindible por cuatro motivos (FEMP, 2008):

- Porque en la elaboración del producto turístico se dan cita elementos de producción pública junto con elementos de producción privada (prestadores de servicios, propiedad de recursos).

- Porque en la gestión integral de la cadena de valor, necesaria para conseguir experiencias turísticas satisfactorias, intervienen la iniciativa privada y la pública. 
- Porque es necesario alinear los objetivos políticos, sociales y económicos que se le asignan a la actividad turística y que determinan y gestionan distintos agentes.

- Porque la imagen global de un destino depende crucialmente de su imagen turística, lo que exige compartir visiones, estrategias y recursos.

Una pieza clave para la articulación de un marco de cooperación estable son las organizaciones que agrupan a los actores. Los diversos agentes del sistema turístico pueden agruparse y colaborar a través de diferentes estructuras. No existe acuerdo sobre qué categorías engloba la idea de instituciones de cooperación, pero las que aparecen con más frecuencias son: las redes de participación, coordinación y cooperación (tres conceptos sobre los que no termina de existir un consenso); los partenariados o las entidades de gestión público-privada, también denominadas Organizaciones de Gestión de Destinos (Destination Management Organizations, en adelante DMOs). Estas últimas son las más institucionalizadas y proveen de los mecanismos adecuados para establecer las interrelaciones entre decisores así como de las fórmulas de colaboración entre los mismos (Sheehan y Ritchie, 2005).

Para la OMT (2004) una DMO es una organización responsable de la organización y marketing de un destino y puede operar a nivel nacional, regional y local. La FEMP (2008), por su lado, define DMO como la responsable de la gestión y comercialización de los destinos pudiendo tener un campo de actuación correspondiente a un área geográfica más o menos amplia, de ámbito nacional, regional o local, entendiendo el concepto destino como un territorio muy concreto y de extensión bastante limitada y perfectamente identificable con un nombre o una marca de destino.

Desde hace algún tiempo los gobiernos locales de destinos turísticos han ido desarrollando distintas estrategias para combatir las profundas dificultades a las que se enfrentan, en especial las dificultades para responder financieramente a las expectativas del sector privado sobre el papel que deberían asumir. La evolución de los órganos creados para el impulso turístico de los municipios ha pasado por varias fases. En el momento actual el acento se pone en la posibilidad de generar estructuras de coordinación entre los diversos agentes que intervienen en el sector turístico. Pero estas estructuras no responden a un solo modelo, más bien al contrario: son el fruto de iniciativas diversas que parten de contextos específicos y presentan una altísima variedad. Cada destino tiene un grado de desarrollo turístico propio, un desarrollo institucional fruto de su propia historia, un sector privado constituido por agentes más o menos innovadores y, por tanto, una cultura colaborativa específica. Así que no existe una fórmula que sea posible aplicar a cualquier destino, sino una alta diversidad organizativa, con distintos modelos que podrían ordenarse en un eje «propuestas tradicionales - propuestas innovadoras».

Las asociaciones entre actores pueden tener nombres y estructuras diferentes y formarse en el sector privado, en el sector público o entre el sector privado y el sector público. Los diferentes tipos de estructuras de colaboración pueden sustentarse en variadas formas: consorcio, sociedad de participación, alianza estratégica, comercialización cooperativa, relación de la cadena de valor, red comercial o subcontratación, en función del nivel de compromiso y funciones que cada uno de los agentes asuma (OMT, 2004). En España existen, según la FEMP (2008) diversos modelos de entidades de gestión público-privada o DMOs: Concejalía, Patronato, EPEL, Sociedad Mercantil, Consorcio, Fundación y Asociación Civil. 
Cualquier reflexión sobre la pertinencia de los diferentes modelos en relación con destinos turísticos concretos precisa de algunas reflexiones previas.

a) Antes de optar por una u otra estructura organizativa sería necesario tomar algunas decisiones sobre las características más relevantes que tendría el futuro órgano. De este modo, el órgano tendría que tener, como punto de partida, las siguientes características (Peters, 1998):

- Concurrencia de la voluntad de al menos dos actores distintos

- Creación de una estructura que no tiene relaciones de autoridad con los participantes que la crean

- Carácter estable de la estructura creada

- Cada actor transfiere recursos, en proporción diferente

- Se asume una responsabilidad conjunta de los participantes en los resultados y el impacto de las actividades

b) En la elección del modelo, es clave la fórmula jurídica que se adapte ya que debe estar vigente durante bastante tiempo. Las dos fórmulas básicas se apoyan en el derecho público o en el derecho privado.

c) Tanto a nivel regional como local, la DMO debería permitir la participación activa de una representación de todos los grupos de interés que interactúan en el destino. Generalmente, el sector privado se siente atraído por la función de comercialización de la DMO, sin embrago, la entidad ha de servir de puente entre las administraciones públicas y la industria en muchos otros ámbitos. De este modo, una DMO o entidad de gestión mixta puede formarse por muy diversas razones y mantener un conjunto de funciones muy diversas. Así, puede establecerse para crear nuevos productos o servicios o desarrollar investigación e innovación, para alcanzar un mayor nivel de eficiencia o aprovechar las economías de escala, para abrir mercados anteriormente inaccesibles o, simplemente, para mancomunar recursos, tanto financieros como humanos. Sin embargo, no existen fórmulas ideales de modelos de gestión, los modelos son fruto de un proceso de adaptación a las circunstancias específicas de cada ciudad (FEMP, 2008).

d) Por lo general, en la decisión de creación de un órgano de gestión turística con participación del sector privado, el punto de partida es que sea el actor público el que traspase la mayor parte de sus funciones al nuevo órgano. Una posición muy diferente a la del sector privado que, no derivando ninguna de sus funciones tradicionales, pasaría a tener competencias decisorias sobre los asuntos que se decidan.

e) La participación del sector privado en una DMO puede adoptar diferentes grados. Según la FEMP (2008), de menor a mayor la participación en la toma de decisiones podría ser de tipo consultivo, minoritario, paritario o mayoritario. Este último, muy poco frecuente, es el sistema de representación por el que optan los destinos en donde la colaboración público privadas está más avanzada. Sería imprescindible una articulación fluida de los representantes del sector privado y una alta profesionalización de los agentes dedicados al turismo en el sector público.

f) Por último, otro de los aspectos de gran importancia en la conformación de una entidad de gestión mixta del destino es la financiación de la organización. La financiación 
predominante en los modelos españoles es pública (FEMP, 2008). De hecho, «por un lado está una gran mayoría de Entes que dependen totalmente (o casi) de la financiación pública, y por otro, un pequeño grupo en que la financiación privada es muy importante y que hacen subir el promedio. Entre éstos destacan Barcelona, Salou, Cuenca, Valladolid y Tenerife» (FEMP, 2008). La financiación básica debería garantizar la estructura organizativa, siendo competencia de los gestores del nuevo órgano garantizar una captación de fondos suficientes como para hacer frente a las exigencias básicas del sector privado, que siempre se relacionan con promoción y marketing.

\section{DISEÑO DE LA INVESTIGACIÓN}

Durante los meses de noviembre de 2009 a junio de 2010 se llevó a cabo una investigación con el objetivo de analizar diferentes indicadores que permitieran diseñar una propuesta de órgano de gestión mixta para el turismo de la ciudad. La hipótesis de partida era que no existe un modelo teórico de órgano de gestión mixta que pueda ser implantado de manera directa en cualquier destino turístico, siendo el contexto específico y sus condiciones los que acaban aconsejando qué tipo de institución de gestión de destino es posible diseñar e implantar. Se considera que para elegir el mejor modelo de órgano de gestión turística para un destino de nivel municipal es necesario investigar tres categorías: caracterización del turismo, importancia relativa del turismo en el destino y madurez colaborativa de los actores turísticos del municipio. Cada categoría se articula en dimensiones y, de estas, se establecen indicadores. En la tabla 1 se pueden ver las categorías, dimensiones, indicadores y fuentes.

\section{Tabla 1 \\ CATEGORÍAS A CONSIDERAR PARA DISEÑAR UNA DMO}

\begin{tabular}{|c|c|c|c|}
\hline Categoría & Dimensión & Indicador & Fuente \\
\hline \multirow[t]{3}{*}{$\begin{array}{l}\text { Caracterización del } \\
\text { turismo }\end{array}$} & Recursos turísticos & $\begin{array}{l}\text { Grado de desarrollo de } \\
\text { recursos y productos } \\
\text { turísticos en destino }\end{array}$ & $\begin{array}{l}\text { - Análisis bibliográfico y } \\
\text { documental } \\
\text { - Análisis de información turística } \\
\text { generada por el propio destino } \\
\end{array}$ \\
\hline & $\begin{array}{l}\text { Comportamiento del } \\
\text { turista/visitante en destino }\end{array}$ & $\begin{array}{l}\text { Grado de homogeneidad/ } \\
\text { heterogeneidad en la visita }\end{array}$ & $\begin{array}{l}\text { - Análisis bibliográfico y } \\
\text { documental }\end{array}$ \\
\hline & Oferta turística & $\begin{array}{l}\text { Diversidad de la oferta } \\
\text { turística } \\
\text { Grado de madurez de la } \\
\text { oferta turístico }\end{array}$ & $\begin{array}{l}\text { - Análisis de fuentes primarias en } \\
\text { el destino } \\
\text { - Entrevistas semi-estructuradas a } \\
\text { los actores turísticos } \\
\end{array}$ \\
\hline $\begin{array}{l}\text { Importancia del turismo en } \\
\text { el destino }\end{array}$ & $\begin{array}{l}\text { Grado de dependencia del } \\
\text { turismo }\end{array}$ & & $\begin{array}{l}\text { - Análisis bibliográfico y } \\
\text { documental }\end{array}$ \\
\hline \multirow[t]{2}{*}{$\begin{array}{l}\text { Madurez colaborativa de } \\
\text { los actores }\end{array}$} & $\begin{array}{l}\text { Experiencia de las } \\
\text { instituciones públicas }\end{array}$ & $\begin{array}{l}\text { Existencia de algún órgano } \\
\text { de administración turística } \\
\text { previo }\end{array}$ & $\begin{array}{l}\text { - Análisis de fuentes primarias en } \\
\text { el destino } \\
\text { - Entrevistas semi-estructuradas a } \\
\text { los actores turísticos } \\
\end{array}$ \\
\hline & $\begin{array}{l}\text { Experiencia previa de } \\
\text { colaboración }\end{array}$ & & $\begin{array}{l}\text { - Análisis de fuentes primarias en } \\
\text { el destino } \\
\text { - Entrevistas semi-estructuradas a } \\
\text { los actores turísticos }\end{array}$ \\
\hline
\end{tabular}

Fuente: Elaboración propia. 
Como fuentes primarias se consultó la documentación existente en el Ayuntamiento y se utilizaron los datos estadísticos disponibles. Al utilizar fuentes diversas se optó por escoger el año 2008 como año de referencia para la toma de datos de caracterización, ya que es el último año del que existen datos basados en encuestas de visitantes y un estudio sobre comportamiento turístico en la ciudad ${ }^{2}$.

Además, se realizaron 22 entrevistas semi estructuradas a actores relacionados con el turismo de la ciudad ${ }^{3}$. El guión de la entrevista se reproduce en la tabla 2.

Tabla 2

GUIÓN ENTREVISTA A ACTORES

Introducción: explicación del proyecto y caracterización del actor

1. Cuénteme un poco cuál es su relación con el sector turístico de Aranjuez

Parte 1. Percepción del turismo en Aranjuez hoy y en el futuro

2. ¿Cómo describiría el turismo hoy en Aranjuez?

3. ¿Cómo lo imagina dentro de diez años teniendo en cuenta los cambios al que el sector turístico, en general -no solo en Aranjuez- está sometido?

4. Dígame si está de acuerdo o no con las siguientes afirmaciones:

- $\quad$ El turismo es una dimensión estratégica que siempre resulta aconsejable y efectiva.

- La actual oferta que el turista encuentra en Aranjuez tiene el grado de amenidad suficiente para satisfacer sus expectativas.

- La oferta turística de Aranjuez resulta adecuada y accesible para todos los públicos, incluidos los no instruidos.

- La ciudad, en tanto que destino turístico, debería desarrollar mecanismos de reserva directa que respondan a la apuesta de los turistas por la organización individual de sus viajes.

- Las consecuencias de la insatisfacción del turista son más importantes desde que existe la web 2.0.

Parte 2. Fortalezas y debilidades de Aranjuez como destino turístico

5. ¿Cuáles son, en su opinión, las fortalezas de Aranjuez como destino turístico?

6. ¿Y cuáles son sus debilidades?

7. Dígame si está de acuerdo o no con las siguientes afirmaciones:

- Aranjuez es un destino turístico consolidado.

- El destino turístico debe basarse en la idea de «ciudad histórica»

2 El análisis del sector turístico de Aranjuez parte de una dificultad importante derivada de la falta de información tanto cualitativa como cuantitativa. El municipio no cuenta con un estudio sistemático del número, motivaciones y gasto de los turistas-excursionistas. Los trabajos más sólidos se realizaron durante los años 1999 -2000 (Troitiño et al, 2000) y fueron ampliados en 2003 (García, 2003; Mínguez, 2003). A pesar de que han transcurrido más de diez años, sus conclusiones pueden servirnos de referencia para nuestro estudio.

3 Se entrevistaron a los responsables turísticos del Ayuntamiento (2); a representantes de los tres partidos políticos con concejales (3); al responsable del Museo Taurino (1); a los representantes de asociaciones - Asociación de la Pequeña y Mediana Empresa (APYME); Asociación Empresarial Hostelera (AHERO); Agrupación de Hostelería; Cámara de Comercio e Industria; Asociación de Desarrollo Rural Aranjuez-Comarca de La Vegas (ARACOVE) y Fundación Aranjuez Paisaje Cultural- (5) y a empresarios turísticos (11). 


\begin{tabular}{|cl|}
\hline$-\quad$ El hecho de ser Paisaje Patrimonio de la Humanidad sólo tiene ventajas \\
$-\quad$ Los turistas se llevan una imagen amplia de la ciudad de Aranjuez \\
$-\quad$ Los turistas consumen diferentes productos turísticos cuando nos visitan \\
- $\quad$ En Aranjuez los turistas culturales reciben explicaciones suficientes sobre aquello \\
que es objeto de su visita. \\
Parte 3. Papel que han de asumir los agentes públicos y privados para mejorar la situación \\
8. ¿Qué hace el Ayuntamiento en relación al turismo? \\
9. ¿Qué podría hacer el ayuntamiento para mejorar el destino? \\
10. ¿Qué podrían hacer los empresarios para mejorar el destino Aranjuez? \\
11. ¿Qué podrían hacer juntos? \\
12. ¿Cómo podría usted colaborar? \\
13. Dígame si está de acuerdo o no con las siguientes afirmaciones: \\
- Existen mecanismos en la gestión del destino que aglutinan los intereses de todos los \\
agentes implicados. \\
El sector público debe tomar la iniciativa en el desarrollo turístico de la ciudad. \\
El ayuntamiento y los empresarios mantienen una relación que favorece el \\
aprovechamiento de la potencialidad del destino.
\end{tabular}

Fuente: Elaboración propia.

\section{ANÁliSiS PARA EL DISEÑO DE UN ÓRGANO DE GESTIÓN DE DESTINO. ELEMENTOS OBJETIVOS}

\subsection{Caracterización del turismo}

\subsubsection{Los recursos turísticos de la ciudad}

A nivel urbano, Aranjuez destaca en el contexto nacional por el diseño de su trama urbana: una ciudad barroca creada y concebida para funciones cortesanas que será marco de eventos, festivales y ceremonias relacionadas con la corte. Esto tiene como consecuencia una alta densidad patrimonial. De entre sus muchos elementos patrimoniales, diez ${ }^{4}$ elementos patrimoniales han sido declarados Bienes de Interés Cultural (Registro de B.I.C. del Ministerio de Cultura). Por su parte, los documentos de planeamiento urbano extienden la protección a 25 edificios, catalogados como de interés histórico-artístico en el Plan General de Ordenación Urbana (1996)5. A estos elementos patrimoniales del conjunto urbano hay que añadir los recursos del patrimonio rural, natural y paisajístico: huertas,

4 Iglesia y arcos de San Antonio, Casa de Infantes, Jardín de la Isla, Jardín del Príncipe, Jardín de Isabel II, Casita del Labrador, Real Convento de San Pascual, Palacio de Aranjuez con sus dependencias, Casa de Caballeros y Oficios y Jardines del Brillante y del Deleite.

5 Palacio Real, Casa de Oficios y Caballeros, Casa de Infantes y Casa de Atarfe, Casita del Labrador, Convento de San Pascual, Iglesia de San Antonio, Hospital de San Carlos, Plaza de Toros, Iglesia de Alpajés, Iglesia del Cortijo de San Isidro, Casa de la Monta / Yeguada de Sotomayor, Regimiento de Pavía, Palacio de Godoy, Palacio de Osuna, Palacio de Medinaceli, Palacio de Silvela, Colegio de Huérfanas de la Infantería, Colegio de San Estanislao, Mercado de Abastos, Estación de Ferrocarril, Antiguo Palacio de Godoy, Arcos de la Plaza de San Antonio, Bodega del Cortijo de San Isidro y Teatro. 
sotos, casillas agrícolas, puentes, explotaciones agrarias tradicionales con sus edificaciones anexas, conjunto del Real Cortijo de San Isidro, etc. A estos elementos se suman los elementos de patrimonio industrial, patrimonio arqueológico y patrimonio etnográfico (fiestas y gastronomía).

Aunque la singularidad de Aranjuez se asienta no sobre el valor aislado de sus elementos más monumentales, sino sobre los valores urbanos y paisajísticos del conjunto formado por la ciudad, el palacio, los jardines y la zona agrícola. De hecho, la justificación de la inclusión de Aranjuez en la Lista del Patrimonio Mundial bajo la figura de Paisaje Cultural se basa en que el paisaje de Aranjuez «responde a la suma de relaciones entre la naturaleza y la actividad humana; entre los arroyos sinuosos y el diseño geométrico del paisaje; entre el rural y el urbano; entre el paisaje del bosque y la arquitectura delicada de sus edificios» (UNESCO, 2001).

\subsubsection{El comportamiento turístico}

El comportamiento del turismo de Aranjuez es coherente con su condición de destino turístico patrimonial dentro de la Región de Madrid, siendo el excursionismo la fórmula de viaje mayoritaria. El excursionismo es practicado tanto por los residentes, como por los visitantes a la ciudad de Madrid, siendo ésta el mayor núcleo emisor de visitantes. La visita se encuentra estrechamente relacionada con la condición de Real Sitio y la existencia de diversos elementos patrimoniales asociados.

Es difícil conocer el número de turistas y visitantes que llegan a la ciudad. Para acercarnos a una cifra aproximada consideraremos dos datos: el número de visitantes atendidos y registrados en la oficina de turismo y el número de entradas al Palacio Real.

Las cifras del número de visitantes han evolucionado de 25.656 visitantes atendidos y registrados en la oficina de turismo y registrados en el año 2003, a 73.367 en 2007, y a 110.500 en el año 2009 (Delegación de Turismo, Ayuntamiento de Aranjuez, 2009) ${ }^{6}$.

El Palacio Real y sus inmediaciones -Jardines de la Isla-Parterre- tienen el mayor porcentaje de visitas, tanto individuales como en grupo. De este modo, el porcentaje de visitas individuales a los elementos patrimoniales entre los años 1999-2000 fueron: Palacio Real $(90,4 \%)$, Jardines de la Isla-Parterre $(94,8 \%)$, Jardin del Príncipe (88,7\%), Mercado y Ayuntamiento (56,4\%), Plaza de Toros (17,9\%), Laguna de Ontigola (2,7\%), (Minguez, 2003).

En el año 2009 se registraron 284.456 visitantes al Palacio Real (Patrimonio Nacional, 2010) 7 . Sin embargo, y según los trabajos anteriormente referidos (Troitiño et al, 2000), habría que considerar que un 9,4\% de los visitantes entrevistados no entran en el Palacio, lo cual aumentaría significativamente el número de turistas en el municipio.

Es también relevante el nivel de repetición de la visita a Aranjuez, como en el caso de otros destinos patrimoniales como San Lorenzo del Escorial. En Aranjuez, el 57,3\% de los visitantes lo son por primera vez, pero el $42,7 \%$ que visita la ciudad ya lo había hecho en otras ocasiones (Mínguez, 2007). En cuanto al lugar de procedencia, el $82 \%$ de los visitantes son nacionales, frente al $18 \%$ de los extranjeros. Entre los primeros

\footnotetext{
6 Es necesario señalar que tampoco estas cifras representan el número total de visitas al municipio, si no las que se acercan a la Oficina de Turismo, el Punto de Información o la Furgoneta Turística.

7 En 2013 se registraron 271.355 visitantes al Palacio Real (Patrimonio Nacional, 2013).
} 
Figura 1

PLANO DE DELIMITACIÓN DEL BIEN ARANJUEZ PAISAJE

CULTURAL POR LA UNESCO (2001)

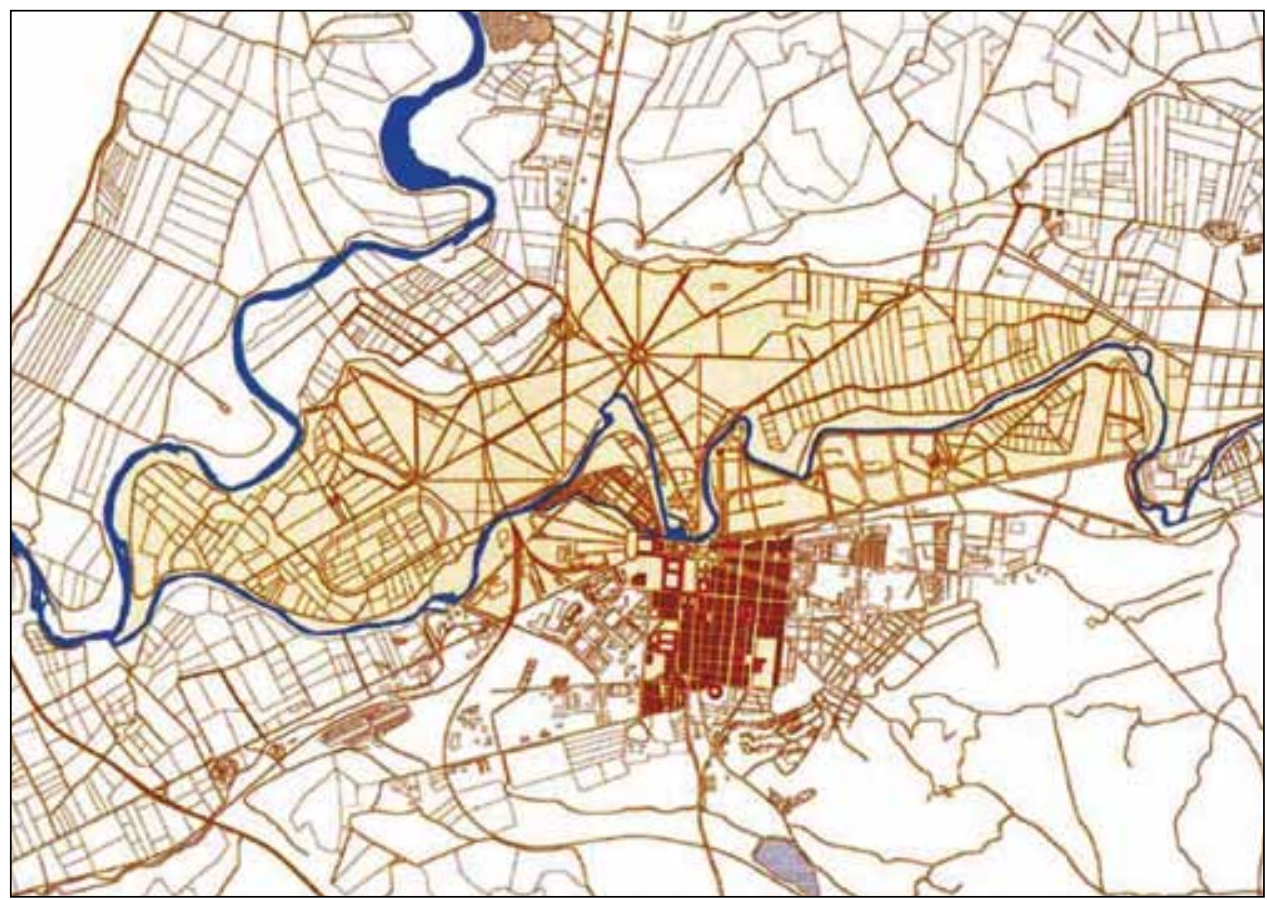

Fuente: http://www.aranjuezcultural.es

destaca la Comunidad Autónoma de Madrid (40,12\%), seguida de Andalucía (9,4\%), Valencia $(9,8 \%)$, Castilla-La Mancha $(7,3 \%)$ y Cataluña $(7,3 \%)$ (Delegación Turismo, Ayuntamiento de Aranjuez, 2009).

\subsubsection{La oferta turística}

Por lo que respecta al número de establecimientos de restauración y de alojamiento, se evidencia una significativa desproporción, lo cual es un indicativo de la elevada tasa de excursionismo de la ciudad. En el año 2009, existían un total de 60 restaurantes con capacidad para atender a algo más de 7.000 comensales en cada turno.

Ese mismo año, el número total de habitaciones era de 567, más 28 bungalow y 179 parcelas en el camping y una casa rural. A esa cifra se llega tras un notable aumento de la planta hotelera desde el año 2000 con la incorporación de dos nuevos hoteles de 4 estrellas - NH y Barceló - que concentran el mayor número de plazas hoteleras de Aranjuez, seguidos por hoteles de menor tamaño y hostales de carácter familiar. Además de hoteles y hostales, la oferta de alojamiento de la ciudad incluye otros tipos de establecimientos como el Camping Internacional de Aranjuez. 
Entre los servicios turísticos de receptivo que ofrecía Aranjuez destaca el Tren de la Fresa, tren histórico cuyo viaje reproduce el recorrido del primer ferrocarril de la Comunidad de Madrid y el segundo de la Península (una iniciativa del Ayuntamiento de Aranjuez en colaboración con el Museo de Ferrocarril de RENFE y Patrimonio Nacional que ha sido recientemente suspendida), el Casino, un campo de golf y nuevos espacios en la ciudad donde se ofrece una amplia oferta para la celebración de congresos, convenciones y reuniones.

La competencia de Aranjuez como destino turístico, en téminos de capacidad alojativa, es media. Otros muncipios equivalentes como destinos turísticos de la Comunidad de Madrid, como Alcalá de Henares, casi le duplica en número de establecimientos, mientras que Chinchón le supera en algunos puntos y El Escorial mantiene más o menos la misma cifra, tal y como muestra la tabla 3.

TABLA 3

NÚMERO DE ALOJAMIENTOS TURÍSTICOS. 1999

\begin{tabular}{|l|c|c|}
\hline & $\begin{array}{c}\text { NÚMERO } \\
\text { ESTABLECIMIENTOS } \\
\text { HOTELEROS }\end{array}$ & NÚMERO HOSTALES \\
\hline Alcalá de Henares & 22 & 8 \\
\hline Aranjuez & 12 & 6 \\
\hline Escorial (EI) & 14 & 2 \\
\hline Chinchón & 16 & 2 \\
\hline
\end{tabular}

Fuente: Instituto Estadística de la Comunidad Autónoma de Madrid, con datos de Dirección General de Turismo. Consejería de Economía y Hacienda

En el caso de las plazas ofertadas, tanto Alcalá de Henares como El Escorial, cuentan con un mayor volumen que Aranjuez, como se ve en la tabla 4.

Tabla 4

NÚMERO DE PLAZAS ALOJAMIENTOS TURÍSTICOS

\begin{tabular}{|l|c|c|c|c|c|c|}
\hline \multicolumn{2}{|c|}{ ARANJUEZ } & \multicolumn{2}{c|}{ EL ESCORIAL } & & \multicolumn{2}{c|}{ ALCALÁ DE HENARES } \\
\hline Año & Dato & & Año & Dato & Año & Dato \\
\hline $\mathbf{2 0 0 4}$ & 1.211 & & $\mathbf{2 0 0 4}$ & 944 & $\mathbf{2 0 0 4}$ & 4.877 \\
\hline $\mathbf{2 0 0 5}$ & 1.546 & & $\mathbf{2 0 0 5}$ & 1.385 & $\mathbf{2 0 0 5}$ & 4.925 \\
\hline $\mathbf{2 0 0 6}$ & 1.546 & $\mathbf{2 0 0 6}$ & 1.645 & $\mathbf{2 0 0 6}$ & 4.925 \\
\hline $\mathbf{2 0 0 7}$ & 1.546 & $\mathbf{2 0 0 7}$ & 1.702 & $\mathbf{2 0 0 7}$ & 5.123 \\
\hline $\mathbf{2 0 0 8}$ & 1.546 & $\mathbf{2 0 0 8}$ & 2.117 & $\mathbf{2 0 0 8}$ & 5.123 \\
\hline
\end{tabular}

Notas: Recoge: Hotel Apartahotel + Hoteles + Hostales + Campamentos + Apartamentos + Pensiones + Casas rurales. Fuente: Instituto Estadística de la CAM, con datos de Dirección General de Turismo. Consejería de Economía y Hacienda, 2009. 


\subsection{Importancia del turismo para el destino: el índice turístico}

Pero, más significativa que las cifras de turismo y de oferta turística de un destino, son los datos que nos permiten comparar el turismo, como sector de actividad del municipio, con otros sectores del mismo. La comparación entre sectores nos permitirá visualizar el grado de dependencia del municipio del sector turístico y, por tanto, la importancia real en términos económicos, laborales y sociales de la actividad.

Para observar este dato utilizaremos los resultados del índice turístico del Anuario de La Caixa que elabora un índice industrial, un índice comercial, un índice de restauración y bares y un índice turístico ${ }^{8}$ para cada municipio de España. Si vemos la tabla 5, observamos que para el municipio de Aranjuez el índice turístico obtiene una cifra menos que los índices industrial, comercial y de restauración y bares. Lo que puede interpretarse como que, a pesar de la importancia relativa que para el municipio pueda tener el turismo, ésta no es tan central como pudiera pensarse por las cifras relacionadas con la propia actividad o, siendo más precisos, el peso relativo de otros sectores en la economía del municipio es mayor. También se han recogido los índices de la ciudad de Madrid y de Alcalá de Henares, para su comparación.

Tabla 5

ÍNDICES ANUARIO LA CAIXA

\begin{tabular}{|l|r|r|r|r|}
\hline $\begin{array}{c}\text { Nombre Municipio } \\
\text { o Total Provincial } \\
\text { y/o CC.AA. }\end{array}$ & \multicolumn{1}{c|}{$\begin{array}{c}\text { Índice } \\
\text { industrial }\end{array}$} & $\begin{array}{c}\text { Índice } \\
\text { comercial }\end{array}$ & $\begin{array}{c}\text { Índice de } \\
\text { restauración y } \\
\text { bares }\end{array}$ & $\begin{array}{c}\text { Índice } \\
\text { turístico }\end{array}$ \\
\hline Madrid & 4.922 & 8.012 & 9.479 & 9.030 \\
\hline Alcalá de Henares & 568 & 377 & 417 & 126 \\
\hline Aranjuez & 144 & 75 & 108 & 46 \\
\hline
\end{tabular}

Fuente: Anuario Económico de España 2009, La Caixa.

Los datos son diferentes si consideramos la dependencia económica del municipio a través del indicador número de personal ocupado. En este caso, y según aparece en la tabla 6, Aranjuez cuenta con una volumen en el sector de hostelería más alto que Alcalá de Henares y El Escorial y menor que el municipio de Chinchón. Esto se debe a la amplitud de la ocupación en el sector de restauración de estos municipios, lo cual refleja de nuevo la importancia del excursionismo frente al turismo. Pero aun así, vemos que el porcentaje de comercio, actividades inmobiliarias o administración pública supera con mucho al de hostelería.

8 El cálculo de los índices se realiza según las siguientes indicaciones: Índice turístico: (Fuente: Anuario Caixa, 2009). 
TABLA 6

NÚMERO DE OCUPADOS POR CADA 1000 HABITANTES EN EL SECTOR SERVICIOS

\begin{tabular}{|l|c|c|c|c|c|c|c|}
\hline & \multicolumn{7}{|c|}{ Ocupados por 1.000 habitantes } \\
\hline & Comercio & Hosteleria & $\begin{array}{c}\text { Transporte y } \\
\text { comunicaciones }\end{array}$ & $\begin{array}{c}\text { Intermediación } \\
\text { financiera }\end{array}$ & $\begin{array}{c}\text { Actividades } \\
\text { inmobiliarias }\end{array}$ & $\begin{array}{c}\text { Administración } \\
\text { Pública }\end{array}$ & $\begin{array}{c}\text { Otros } \\
\text { servicios }\end{array}$ \\
\hline $\begin{array}{l}\text { Comunidad } \\
\text { de Madrid }\end{array}$ & 80,91 & 27,25 & 37,35 & 16,73 & 111,42 & 87,40 & 47,20 \\
\hline & & & & & & & \\
\hline Aranjuez & 58,30 & $\mathbf{2 6 , 6 7}$ & 10,68 & 5,77 & 41,77 & 59,60 & 29,86 \\
\hline $\begin{array}{l}\text { Escorial } \\
\text { (El) }\end{array}$ & 42,51 & 17,13 & 6,43 & 4,33 & 22,37 & 39,22 & 29,36 \\
\hline $\begin{array}{l}\text { Alcalá de } \\
\text { Henares }\end{array}$ & 75,40 & 15,90 & 19,89 & 8,54 & 45,75 & 74,15 & 27,79 \\
\hline Chinchón & 63,11 & 43,54 & 8,99 & 10,19 & 15,78 & 53,33 & 17,97 \\
\hline
\end{tabular}

Fuente: Instituto Estadística de la Comunidad Autónoma de Madrid, con datos de Dirección General de Turismo. Consejería de Economía y Hacienda, 2009.

\subsection{Madurez colaborativa de los actores públicos y privados}

Uno de los rasgos que caracterizan al sector turístico de Aranjuez es la fragmentada y heterogénea naturaleza de los numerosos actores que, de una forma u otra, están involucrados en los procesos de planificación, dirección, comercialización y promoción de esta actividad: agentes directa e indirectamente implicados en turismo procedentes de diferentes sectores socio-económicos (transporte, alojamiento, comercialización, restauración, etc.); actores operando a diferentes niveles (pequeños negocios locales versus cadenas nacionales); organismos públicos; turistas y población local.

\subsubsection{Grado de desarrollo institucional previo de la administración pública turística}

En el municipio de Aranjuez las competencias en materia de turismo han compartido espacio institucional con otras materias. Los temas de turismo se han gestionado tradicionalmente junto a los asuntos de desarrollo económico y empleo. Este modelo organizativo ha tenido algunas ventajas, la más evidente es que ha permitido observar la actividad desde la perspectiva del desarrollo global de la ciudad, pero también desventajas, fundamentalmente que el turismo no ha tenido nunca un aparato administrativo propio y esto, evidentemente, puede repercutir negativamente en el sector.

La falta de un órgano autónomo y estable no ha permitido el desarrollo de un espacio institucional propio con experiencia acumulada. La oportunidad más clara se perdió con el Plan de Dinamización Turística que se desarrolló desde 1998 hasta el 
año 2000 y en el que se invirtieron 450 millones de pesetas (más de dos millones y medio de euros). Los objetivos del plan fueron: aprovechar todas las potencialidades turísticas de Aranjuez; mejorar el medio urbano y natural; articular recursos y ofertas turísticas a partir de la creación de órganos de coordinación y participación en el diseño de estrategias de producto, precio, promoción y comercialización. Ya entonces se pensó en la necesidad de articular estrategias de coordinación, pero nunca se avanzó en dicho sentido. Tampoco nunca se ha creado una concejalía específica o un órgano institucional con mayor peso político administrativo.

La única iniciativa que funciona desde junio de 2005 es el Consejo Local de Turismo. Formado por representantes del mundo de la hostelería, el sector hotelero, la Cámara de Comercio, empresas turísticas, la universidad, técnicos del Ayuntamiento, Patrimonio Nacional, los partidos políticos y los sindicatos, su objetivo era promover la presencia de Aranjuez en los mercados turísticos nacionales e internacionales, potenciar los recursos turísticos existentes, proponer nuevas medidas e iniciativas y asesorar al Ayuntamiento de Aranjuez en estos temas. El Consejo ha venido funcionado desde entonces, pero su falta de estructura organizativa estable y su carácter consultivo han de ser superados por una opción organizativa más avanzada.

La falta de institucionalidad permitía observar también una consecuencia clara en la falta de estabilidad del personal dedicado a trabajar en turismo desde el ayuntamiento. De hecho, hasta el año 2008 ni siquiera existía en el municipio un puesto de técnico de turismo.

\subsubsection{Grado de colaboración institucional previa por parte del empresariado turístico}

El grado de madurez del sector privado es otro indicador clave para determinar si Aranjuez estaría preparado para conformar una entidad mixta de gestión del destino. En este sentido, no se juzga ni la mayor o menor eficacia empresarial de las distintas unidades de negocio, ni su mejor o peor sistema de gestión, ni, por supuesto, sus resultados empresariales. Se hace referencia exclusivamente a la capacidad que podrían desarrollar con objeto de implicarse en un órgano de gestión turística mixta especialmente en términos de posible co-financiación y aportación de recursos de otro tipo, partiendo de las opiniones que los propios actores han manifestado.

Sin embargo es importante analizar la percepción que el conjunto de agentes tiene sobre este grupo de interés (sector privado). Se trataría de un elemento definitivo en el proceso de determinación de las posibilidades de incorporar una entidad de gestión mixta o DMO.

En este sentido, el empresariado turístico de Aranjuez se caracteriza por: (a) un fuerte individualismo, (b) la consideración de que debe ser la administración quién asuma y lidere cualquier proceso innovador de trabajo conjunto, (c) débil visión de largo plazo, (d) escasas iniciativas de innovación para la creación de nuevos productos, (e) una necesidad de mejora formativa, así como (f) una cultura local con poca orientación global.

El individualismo que manifiesta el sector empresarial se refleja en la debilidad de las asociaciones, las cuales no consiguen motivar al empresariado lo suficiente como para generar un programa continuado de reuniones y actuaciones que las convierta en 
claros interlocutores. Gran parte del sector percibe como una situación de enfrentamiento permanente con la administración la falta de capacidad de ésta para gestionar ayudas inmediatas a las empresas. La debilidad de una visión estratégica y de largo plazo se observa en la concepción del resto de las empresas turísticas como competencia, en lugar de intentar trabajar conjuntamente. Son conscientes de las debilidades de Aranjuez como destino turístico, sin embargo, no plantean opciones de desarrollo que impliquen un cambio en el sector privado, permaneciendo inmóviles frente al conjunto.

La innovación es uno de los pilares básicos para el crecimiento y el desarrollo turístico en Aranjuez, pero la creación de nuevos productos, necesidad de primer orden, debe partir de los empresarios. Las entidades turísticas deben ser las verdaderas promotoras de los productos por su capacidad de gestión así como por su conocimiento del cliente y sus necesidades.

Sería importante mejorar la capacitación y participación del empresariado en cuestiones que permitan trasformar la visión tradicional del gestor hacia un concepto ligado al desarrollo turístico de la ciudad. Resulta preciso un cambio en las sensibilidades existentes hacia conceptos como la nueva competitividad, que debería acercarse a las nociones de cooperación (co-opetitividad). Esto, unido a la mejora del funcionamiento de las asociaciones como mecanismos reales de defensa de los intereses de las empresas de Aranjuez, ayudaría en el proceso de creación de productos conjuntos, a través de acuerdos empresariales, que conformaran una oferta turística más diversificada para el destino.

\section{PROPUESTA PARA EL DISEÑO DE UN ÓRGANO DE GESTIÓN MIXTA}

\subsection{Percepción de los agentes públicos y privados de la situación del sector turístico en Aranjuez}

Del análisis del discurso de las entrevistas con actores turísticos relevantes del sector público y del privado se extraen cuestiones de gran interés.

- Se califica al turismo de Aranjuez como un sector de alta potencialidad pero cuyas circunstancias actuales permiten definirlo con los términos de «frágil»y «débil».

- Los actores públicos y privados entienden que la situación actual del turismo en el municipio es débil fundamentalmente en relación con duración de la estancia (poca pernoctación), capacidad de gasto y tipología del cliente (segmento de demanda de categoría económica media-baja).

- Las razones a las que se recurre para explicar la fragilidad del destino son la baja oferta de productos turísticos, a excepción del palacio y los jardines, la falta de oferta complementaria, así como la carencia de iniciativas para la puesta en valor del conjunto de elementos existentes.

- Se señala la falta de una política turística decidida de la administración local como uno de los factores clave. Se insiste en que las acciones en materia de turismo impulsadas por el ayuntamiento son casi inexistentes, débiles y faltas de continuidad, inversión y capacidad de contratación de recursos humanos cualificados. La improvisación y la falta de un plan estratégico que oriente las actuaciones en materia de política turística es otro de los aspectos destacados. 
- Los actores entienden que el turismo de Aranjuez precisa de sinergias. La colaboración ha de reflejarse desde la administración tanto vertical (con la CAM y Patrimonio Nacional) como horizontalmente (con otras concejalías), entre los empresarios y el sector público, e incluso cooperando con otros municipios del área.

- Tampoco es muy positiva la visión del empresariado. Agentes de distinta procedencia lo califican de individualista. Las empresas turísticas tienen vocación de corto plazo y trabajan en muy pocas ocasiones de manera colaborativa, lo que repercute en el resultado final, haciendo que las iniciativas sean pocas y no exista innovación de productos y/o actividades puestas a disposición de la demanda.

- La visión que distintos actores tienen acerca del futuro del turismo en Aranjuez es de estancamiento, incluso de retroceso en algunos aspectos. La perspectiva es pesimista si el sector público y el privado no asumen sus debilidades. El municipio precisa de un cambio de rumbo en el que se aúnen esfuerzos de todos los agentes y comiencen a ponerse en valor todos los recursos de los que se dispone orientándose a un turismo de calidad.

No obstante, para analizar las posibilidades de implantación de una entidad de gestión mixta del turismo en Aranjuez, capaz de mejorar el desarrollo turístico del municipio, es importante conocer las opiniones de los actores al respecto de su interés como grupo, así como de la imagen que tienen del resto de agentes con los que colaborarían.

En este sentido, el papel que debería asumir el sector público, según el conjunto de grupos de interés, es el de tomar la iniciativa del desarrollo turístico de la ciudad. La percepción de los agentes, tanto de las organizaciones privadas, como de las asociaciones civiles y empresariales, manifiesta un cierto grado de desconfianza hacia los actores públicos pues entienden que la administración realiza pocas acciones en materia de turismo, muchas de las cuales no son siquiera las adecuadas. Las razones de la citada pérdida de confianza son la fragilidad y debilidad de la administración dado el bajo presupuesto asignado a turismo, la falta de recursos humanos cualificados en este ámbito y la falta de acciones vertebradas y sostenidas en el tiempo. Esta situación provoca la falta de un plan estratégico que contemple unos objetivos y unas acciones concretas y tangibles en materia turística así como una visión a largo plazo que logre establecer una línea de trabajo homogénea y competitiva para el destino.

Uno de los principales inconvenientes de la administración es viene de la falta de comunicación con los agentes implicados. Si bien, la superación de esta situación es una necesidad de primer orden para el sector público, el sector privado percibe una carencia importante en este sentido. De este modo, los actores de Aranjuez podrían dividirse en, aquellos que no conocen las actividades de la administración, por falta de información de la misma o por desinterés del propio agente, y los que entienden que se está haciendo un esfuerzo por escuchar a los distintos grupos de interés pero ni los mecanismos son los adecuados ni se produce un seguimiento de los compromisos acordados.

Las peticiones de los distintos actores locales en Aranjuez se traducen en la tabla 7, donde se especifican las debilidades funcionales detectadas por los agentes así como las acciones o programas de actuación que los grupos entienden prioritarios de cara al desarrollo turístico y, por tanto, demandan a la administración para su incorporación al conjunto de funciones públicas en materia turística. 
Tabla 7

PERCEPCIÓN DE LOS ACTORES ACERCA DE LOS PROGRAMAS DE ACCIÓN NECESARIOS A REALIZAR POR LA ADMINISTRACIÓN PÚBLICA DE ARANJUEZ

\begin{tabular}{|c|c|c|}
\hline AGENTE & DEBILIDADES FUNCIONALES & $\begin{array}{c}\text { PROGRAMAS DE ACCIÓN } \\
\text { NECESARIOS }\end{array}$ \\
\hline $\begin{array}{l}\text { Empresas } \\
\text { hoteleras }\end{array}$ & $\begin{array}{l}\text { - Poca promoción } \\
\text { - Falta de productos turísticos } \\
\text { - Debilidad en infraestructuras }\end{array}$ & $\begin{array}{l}\text { - Programa de promoción (continuado, } \\
\text { consensuado, diferenciado por mercados } \\
\text { y de mayor presupuesto) } \\
\text { - Programa de creación de nuevos } \\
\text { productos } \\
\text { Programa de mejora y modernización de } \\
\text { las infraestructuras }\end{array}$ \\
\hline $\begin{array}{l}\text { Empresas de } \\
\text { restauración }\end{array}$ & $\begin{array}{l}\text { - Poca promoción } \\
\text { - Desinterés y no atención a } \\
\text { las necesidades propias de la } \\
\text { restauración (más flexibilidad) } \\
\text { - Déficit de actuaciones para poner } \\
\text { en valor la huerta de Aranjuez }\end{array}$ & $\begin{array}{l}\text { Programa de promoción (continuado, } \\
\text { consensuado, diferenciado por mercados } \\
\text { y de mayor presupuesto) } \\
\text { - Programa de puesta en valor de la } \\
\text { huerta } \\
\text { - Programa de incentivos a empresarios } \\
\text { turísticos }\end{array}$ \\
\hline $\begin{array}{l}\text { Empresas } \\
\text { turismo } \\
\text { receptivo }\end{array}$ & $\begin{array}{l}\text { - Falta de producto turístico } \\
\text { - Poca promoción } \\
\text { - Escasa oferta complementaria de } \\
\text { calidad } \\
\text { - Déficit de actuaciones para atraer } \\
\text { un público objetivo con mayor } \\
\text { capacidad de gasto }\end{array}$ & $\begin{array}{l}\text { - Programa de creación de nuevos } \\
\text { productos } \\
\text { - Programa de promoción (continuado, } \\
\text { consensuado, diferenciado por mercados } \\
\text { y de mayor presupuesto) }\end{array}$ \\
\hline $\begin{array}{l}\text { Asociaciones } \\
\text { locales }\end{array}$ & $\begin{array}{l}\text { - Acciones en materia de turismo } \\
\text { sin continuidad ni contenido de } \\
\text { MP/LP } \\
\text { - No existencia de un plan } \\
\text { estratégico de turismo } \\
\text { - Falta de presupuesto y RRHH } \\
\text { cualificados } \\
\text { - Poca valoración de la ciudad } \\
\text { como parte del Patrimonio de la } \\
\text { Humanidad y Paisaje Cultural } \\
\text { - Necesidad de mecanismos de } \\
\text { trabajo conjunto con los agentes } \\
\text { locales }\end{array}$ & $\begin{array}{l}\text { - Plan estratégico (con medidas y } \\
\text { actuaciones concretas y de medio plazo } \\
\text { y largo plazo, así como acciones de } \\
\text { seguimiento) } \\
\text { - Programa de mejora de los rrhh } \\
\text { (cualificación y formación) } \\
\text { - Programa de captación de fondos } \\
\text { (internos a la administración y } \\
\text { extraordinario s procedentes de distintos } \\
\text { agentes) } \\
\text { - Programa de cooperación } \\
\text { (interadministrativa, público-privada y } \\
\text { entre instituciones como Patrimonio de la } \\
\text { Humanidad) } \\
\text { Programa de concienciación ciudadana } \\
\text { (fundamentalmente enfocado al } \\
\text { conocimiento de todos los agentes } \\
\text { locales de las ventajas de ser parte del } \\
\text { Patrimonio de la Humanidad) }\end{array}$ \\
\hline
\end{tabular}




\begin{tabular}{|l|l|l|}
\hline \multicolumn{1}{|c|}{ AGENTE } & DEBILIDADES FUNCIONALES & \multicolumn{1}{c|}{$\begin{array}{c}\text { PROGRAMAS DE ACCIÓN } \\
\text { NECESARIOS }\end{array}$} \\
\hline \multirow{4}{*}{$\begin{array}{l}\text { Actores } \\
\text { políticos } \\
\text { locales }\end{array}$} & $\begin{array}{l}\text { - Desorganización interna de } \\
\text { la administración }\end{array}$ & $\begin{array}{l}\text { o Programa de organización interna } \\
\text { y asignación de responsabilidades } a \\
\text { técnicos cualificados }\end{array}$ \\
& $\begin{array}{l}\text { Poca asignación } \\
\text { presupuestaria a turismo } \\
\text { - Falta de un plan estratégico } \\
\text { de turismo }\end{array}$ & $\begin{array}{l}\text { Programa de cooperación } \\
\text { (interadministrativa, público- } \\
\text { privada y entre instituciones como } \\
\text { Patrimonio de la Humanidad) }\end{array}$ \\
& $\begin{array}{l}\text { Desatención a las } \\
\text { necesidades de los actores } \\
\text { locales. }\end{array}$ & \\
\hline
\end{tabular}

Fuente: Elaboración propia a partir de la información procedente de las entrevistas.

\section{2. Ámbitos en los que podrían trabajar de manera conjunta}

A pesar de la caracterización anterior, el conjunto de actores turísticos del municipio reflejan un elevado grado de interés en la idea de la creación de una entidad de gestión público-privada. Para ello abren las puertas de sus negocios o asociaciones, aunque no ven claro cuáles serían las funciones de la misma, su financiación y el grado de operatividad en la implantación de proyectos reales.

Los actores perciben distintas áreas y formas de colaboración en función de sus características o de la experiencia que hayan tenido en otras entidades o agrupaciones asociativas. Así, un primer grupo (a) apuesta por la colaboración prioritariamente en materia de promoción, otro (b) por las acciones conjuntas para la creación de productos innovadores y de calidad, un tercero (c) requiere y solicita flexibilidad en las propuestas de cooperación para que lleguen a implantarse y sean beneficiosas para el conjunto (proponen la contratación de un técnico independiente) y, el último (d) entiende que previo a la creación de la entidad habría de generarse un clima de mayor confianza entre actores a través de foros o reuniones periódicas.

El nivel de colaboración público-privada más amplio se lo plantean fundamentalmente las asociaciones empresariales y otras locales, los grupos políticos y la propia administración, los cuales continúan en la idea de formular un plan estratégico participativo para el turismo de Aranjuez. Respecto de las posibilidades de colaboración entre actores en el marco de la entidad mixta, muy pocos están dispuestos a poner dinero pero exigen que las acciones sean rentables para ellos, otros creen que deben aportar sus ideas y conocimiento del cliente, la mayoría considera que su mayor aportación es su experiencia, tener un cliente satisfecho y promocionar a través de su empresa la marca Aranjuez y todos están dispuestos a sentarse y trabajar conjuntamente. Las asociaciones, por su lado, se comprometen a mover a sus asociados y motivarles. Así, las áreas de posible colaboración en la entidad, según cada grupo de interés serían las que muestra la tabla 8 . 


\section{Tabla 8}

\section{ÁREAS PREVISIBLES DE COLABORACIÓN EN UNA DMO PARA EL TURISMO EN ARANJUEZ}

\begin{tabular}{|c|c|}
\hline AGENTE & $\begin{array}{c}\text { APORTACIONES PREVISIBLES DE CADA ACTOR } \\
\text { A UN MARCO DE COLABORACIÓN }\end{array}$ \\
\hline Empresas hoteleras & $\begin{array}{l}\text { - Promoción de Aranjuez a través de la imagen de marca del } \\
\text { establecimiento } \\
\text { - Conocimiento de las necesidades del cliente actual que } \\
\text { pernocta en Aranjuez. } \\
\text { - Capacidad de generar ideas y trasmitir experiencias. } \\
\text { - Ofertar alojamientos de calidad } \\
\text { - Trabajar de manera colaborativa con el resto de empresas } \\
\text { turísticas y la administración en acciones de promoción y } \\
\text { de creación de productos conjuntos }\end{array}$ \\
\hline $\begin{array}{l}\text { Empresas de } \\
\text { restauración }\end{array}$ & $\begin{array}{l}\text { - Promoción de Aranjuez a través de la imagen de marca del } \\
\text { establecimiento. } \\
\text { - Fomento de la huerta de Aranjuez a través de su expresión } \\
\text { en la gastronomía. } \\
\text { - Conocimiento de las necesidades del cliente actual. } \\
\text { - Capacidad de generar ideas y trasmitir experiencias. }\end{array}$ \\
\hline $\begin{array}{l}\text { Empresas turismo } \\
\text { receptivo y oferta } \\
\text { complementaria }\end{array}$ & $\begin{array}{l}\text { - Conocimiento del mercado en origen. } \\
\text { - Generando productos turísticos locales innovadores. } \\
\text { - Promocionando Aranjuez a través de los productos } \\
\text { comercializados. } \\
\text { - Participando en foros/reuniones. }\end{array}$ \\
\hline $\begin{array}{l}\text { Asociaciones } \\
\text { empresariales }\end{array}$ & $\begin{array}{l}\text { - Formación y asesoramiento a empresarios. } \\
\text { - Patrocinio de algunas actividades concretas. } \\
\text { - Acciones de sensibilización y motivación al empresariado. } \\
\text { - Participación en foros/reuniones. }\end{array}$ \\
\hline $\begin{array}{l}\text { Otras Asociaciones } \\
\text { locales }\end{array}$ & $\begin{array}{l}\text { - Formación y sensibilización integral de la potencialidad } \\
\text { de los recursos patrimoniales de Aranjuez. } \\
\text { - Visión más amplia y estratégica del destino. } \\
\text { - Organización de seminarios y eventos. } \\
\text { - Participación en foros/reuniones. }\end{array}$ \\
\hline Actores políticos locales & $\begin{array}{l}\text { - Participando en todos los foros en que se nos convoque } \\
\text { - Transmitiendo las necesidades reales del sector turístico } \\
\text { de Aranjuez a través del contacto permanente con la } \\
\text { industria. }\end{array}$ \\
\hline
\end{tabular}

Fuente: Elaboración propia a partir de la información procedente de las entrevistas. 


\section{CONCLUSIONES SOBRE LA VIABILIDAD DE UN ÓRGANO DE GESTIÓN MIXTA PARA EL TURISMO EN ARANJUEZ}

Primera. El papel de los agentes sociales ha cambiado profundamente en los últimos años y este cambio también puede observarse cuando se reflexiona sobre el desarrollo de destinos turísticos locales. En el momento actual se exige la colaboración de todos los agentes implicados en el sistema turístico, mediante el diseño e implantación de estrategias adaptadas a sus realidades pero que implican la creación de marcos institucionales estables que facilitaran la tarea del trabajo común.

Segunda. Una de las herramientas básicas para el trabajo en común es la creación de una institución de gestión de carácter mixto responsable del desarrollo de algunas de las dimensiones relacionadas con el destino globalmente considerado. Existen ya experiencias exitosas de colaboración que, con dimensiones muy distintas, han conseguido dinamizar destinos y cambiar la forma de trabajar por el desarrollo turístico en el nivel local.

Tercera. Para que el esfuerzo en la creación de una institución de carácter mixto tenga resultados positivos es imprescindible analizar el contexto específico, las características del destino turístico y la cultura cooperativa que impera en el mismo. Sólo así podremos determinar las posibilidades y los límites de este tipo de institución, ya que no existe un modelo teórico que pueda ser implantado de manera directa.

Cuarta. Los indicadores que deben ser observados son la dependencia real del municipio respecto del turismo, para determinar el grado de necesidad real que existe por modificar las formas de gestión del turismo. El desarrollo institucional previo de los agentes públicos en materia de turismo, para conocer su experiencia, el liderazgo anteriormente asumido y la credibilidad de sus propuestas. El grado de madurez del sector privado turístico, para conocer cuál es el perfil real de los agente privados, su vocación de colaboración y sus expectativas respecto de lo que puede hacerse desde una plataforma público-privada. Y, finalmente, las áreas que podrían ser entendidas por ambos sectores como posibles espacios de trabajo conjunto.

Quinta. Respecto del análisis del caso concreto, centrado en el municipio de Aranjuez, las principales conclusiones son:

- La apuesta por la calidad en el turismo de Aranjuez pasa por la consideración del municipio como un destino de gran valor cultural y con la suficiente oferta complementaria como para adaptarse a los nuevos requerimientos de la demanda, guiando las iniciativas hacia un cambio en el tipo de turista actual y transformando el mercado de atracción en un segmento instruido, interesado por los aspectos culturales, con capacidad de gasto más alta, menor nivel de intermediación y procedente de municipios cercanos, como Madrid y Toledo (tanto residentes, como turistas culturales de estas ciudades).

- Esta oferta de calidad, por la que habría de apostarse de cara al futuro, debe seguir teniendo como referente básico la capacidad de atracción real del patrimonio formado por palacio y jardines, por un lado, y por la consideración de Aranjuez como Paisaje Cultural Patrimonio de la Humanidad por otro. Estos aspectos han de 
materializarse en un futuro de manera que se conviertan en elementos de primera categoría y logren posicionarse en el mercado como generadores de una demanda con las características definidas anteriormente. Para ello, la colaboración con Patrimonio Nacional es esencial, sin embargo, la concienciación de los actores internos acerca de la importancia de estos recursos es una de las acciones prioritarias en el corto plazo.

- Respecto del papel del sector privado, de cara a la concepción del futuro del turismo en Aranjuez, es necesario que las empresas turísticas mantengan una visión de largo plazo, incorporen productos innovadores a la oferta turística del municipio y formen parte de asociaciones fuertes que les permitan trabajar conjuntamente como dinamizadores de productos turísticos. Sin embargo, para que el turismo en Aranjuez tenga un futuro exitoso y competitivo, la administración pública ha de motivar a los empresarios a través de la gestión de espacios de comunicación que le permitan conocer las iniciativas conjuntas y generar mecanismos para su consideración en el marco del desarrollo turístico.

- El análisis de los indicadores previos aconsejaban un plan de acción en materia de colaboración turística a desarrollar en varias fases. La primera, basada en la creación de una figura organizativa modesta que pudiera ampliar el grado de conocimiento mutuo y confianza entre ambos sectores, con poca competencia, bien definidas y con objetivos claramente consensuados. En una segunda fase podría ampliarse el nivel de acuerdo y diseñar algunas instituciones más abiertas con competencias más amplias.

\section{BIBLIOGRAFÍA}

BEVIR, M. (2011): The Sage Handbook of Governance. London. Sage.

BÖRZEL, T.A. (1998): «Organizing Babylon: on the different conceptions of policy networks», Public Administration, 76 (2): 253-273.

BRAMWELL, B. y LANE, B. (1999): «Collaboration and Partnerships for Sustainable Tourism», Journal of Sustainable Tourism, vol. 7, pp. 179-181.

CAIXA, La (2009) Anuario Económico de España. [Consultado en: http://www.anuarioeco. lacaixa.comunicacions.com]

DREDGE, D. (2006): «Policy networks and the local organisations of tourism», Tourism Management, vol. 27, $\mathrm{n}^{\circ}$ 2, pp. 269-281.

DREDGE, D. y PFORR, C. (2008): «Policy Networks and Tourism governance», en Network Analysis and Tourism. From Theory to Practice. Scott, N., Baggio, R. y Cooper, C. Channel View Publications, Toronto.

FEMP (2008): Modelos de Gestión Turística Local. FEMP. Madrid

GARCÍA HERNÁNDEZ, M. (2003): Turismo y conjuntos monumentales: capacidad de acogida turística y gestión de flujos de visitantes. Tirant lo Blanch. Valencia. 541 pp

JAMAL, T.B. y GETZ, D. (1995): «Collaboration theory and community tourism planning.» Annals of Tourism Research, vol. 22(1), pp. 86-204.

MERINERO RODRÍGUEZ, R. (2009): «Redes de actores y desarrollo turístico. Análisis de casos españoles», Papers de Turisme, $\mathrm{n}^{\circ}$ 46, pp. 57-77. 
MERINERO RODRÍGUEZ, R. y PULIDO FERNÁNDEZ, J.I. (2009): «Desarrollo turístico y dinámica relacional. Metodología de análisis para la gestión activa de destinos turísticos», Cuadernos de Turismo, no 23, pp. 173-193.

MÍNGUEZ GARCÍA, M.C. (2003): «El Real Sitio de Aranjuez: Destino de Excursionismo de la Región turística madrileña». En La actividad turística española en 2002. Editorial Ramon Areces.

MUÑOZ, A. (2008): «Fomento institucional de la cooperación público-privada como factor clave del desarrollo turístico». Segundo Congreso Internacional sobre Turismo y Ocio. Eumed.

NORTH, D. C. (1990): Institutions, Institutional Change and Economic Performance. Cambridge, Cambridge University Press.

OMT (2001): Cooperación entre los Sectores Público y Privado. Para una Mayor Competitividad del Turismo. OMT. Madrid.

OMT (2004): Cooperación y asociaciones en turismo: Una perspectiva mundial, OMT, Madrid.

PAVLOVICH, K. (2008): «Network governance and connectivity: A case study.»Network analysis and tourism: From theory to practice, pp. 79-95.

PECHLANER, H. (2013): «Towards a comprehensive view of tourism governance: Relationships between the corporate governance of tourism service firms and territorial governance». International journal of globalisation and small business.

PETERS, G. B. (1998): «With a Little Help From Our Friends: Public-Private Partnerships as Institutions and Instruments». In: Jon Pierre (Ed.), Partnerships in Urban Governance. European and American Experience. London, McMillan, pp. 11-34.

REED, M. G. (1999): Collaborative Tourism Planning as Adaptative Experiments in Emergent Tourism Setting, Journal of Sustainable Tourism, vol. 7(3), pp. 331-355.

SCOTT, N., BAGGIO, R. y COOPER, C. (2008): Network Analysis and Tourism. From Theory to Practice. Channel View Publications, Toronto.

SCOTT, N., COOPER, C., y BAGGIO, R. (2008): «Destination Networks», Annals of Tourism Research, vol. 35(1), pp. 169-188.

SHEEHAN, L. y RITCHIE, B (2005): «Destination stakeholders. Exploring identity and salience», Annals of Tourism Research, vol. 32, (3), pp. 711-734.

SELIN, S. (1999): «Developing a Typology of sustainable Tourism Partnerships», Journal of Sustainable Tourism, vol. 7, (3 y 4), pp. 260-273.

SELIN, S. y CHAVEZ, D. (1995): «Developing an Evolutionary Tourism Partnership Model», Annals of Tourism Research, vol. 22, (4), pp. 844-856.

SELIN, S. y MYERS, N. (1998). «Tourism marketing alliances: Member Satisfaction and effectiveness attributes of a regional initiative», Journal of Travel and Tourism Marketing, vol. 7, (2), pp. 79-94.

TIMOTHY, D. J. (1998): «Cooperative tourism planning in a developing destination», Journal of Sustainable Tourism, 6(1), pp. 52-68.

TORFING, J., PETERS, B.G., PIERRE, J. y SORENSEN, E. (2012): Interactive Governance: Advancing the Paradigm. Oxford: Oxford University Press.

TROITIÑO VINUESA, M.A.; CALLE VAQUERO, M. de la; GARCÍA HERNÁNDEZ, M. (2000): «Las ciudades históricas como destinos turísticos: patrimonio cultural, 
tipos de visitantes y sistema de acogida local». En La actividad turística española en 1999. Asociación Española de Expertos Científicos en Turismo. Madrid. pp. 545-556.

UNESCO (2001): Declaración de Aranjuez Paisaje Cultural de la Humanidad [Consultado en: http://whc.unesco.org/es/list/1044]

VELASCO GONZÁLEZ, M. (2013): «Gestión pública del turismo. La Gobernanza». En Gestión estratégica sostenible de destinos turísticos, Coord. Juan Ignacio Pulido, Universidad Internacional de Andalucía. 